\title{
Searching for Dark Matter through Vector Boson Fusion Topology at the LHC
}

\author{
Santiago Duque-Escobar, Daniel Ocampo-Henao, and José David Ruiz-Álvarez \\ Instituto de Física, Universidad de Antioquia, A.A. 1226 Medellín, Colombia
}

\begin{abstract}
We describe different searches that have been conducted in the LHC using the vector boson fusion topology, specially in the regard of beyond standard model physics and dark matter. We finalize proposing a first glimpse into what could be a proposal for new searches of dark matter using the vector boson fusion topology with simplified dark matter models.
\end{abstract}

Keywords: vector boson fusion, dark matter, LHC, simplified models

DOI: 10.31526/ ACP.BSM-2021.4

\section{INTRODUCTION}

Searching for a possible dark matter (DM) particle is one of the most interesting and exciting enterprises in particle physics of our days. DM has been, jointly with neutrino masses, become perhaps the most intriguing mystery for particle physics and astrophysics. In our community we tend to accept the existence of DM. Specially as a consideration for building a successful cosmological model. But what is exactly the nature of DM remains hidden among a plethora of models. Is therefore very important to elucidate every possible way of looking for DM.

\section{VECTOR BOSON FUSION AT THE LHC}

Vector boson fusion (VBF) topology has been used in various searches at the LHC experiments, specially ATLAS and CMS. The CMS experiment has been using it for Higgs searches as it is an interesting production channel where the background can be controlled using the VBF characteristics [1]. In this topology the Higgs boson is produced from a vertex with two vectorial bosons from the SM radiated from the incoming quarks in the collisions. Figure 1 depicts the Higgs boson production in the VBF topology at the LHC.

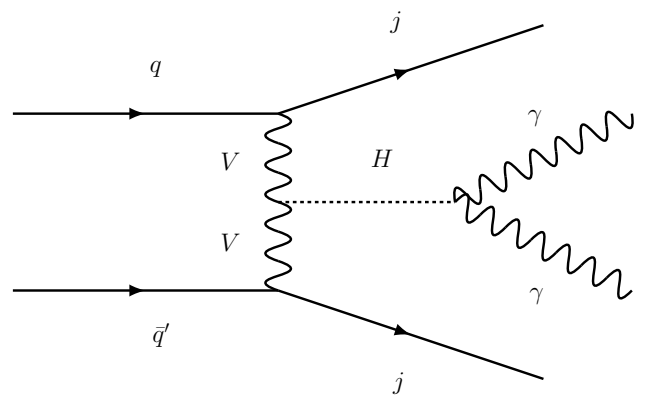

FIGURE 1: VBF topology for SM Higgs production

The outgoing jets from the VBF topology are well separated in $\eta$, their invariant mass is also high and are typically produced in opposite hemispheres of the detector. These characteristic features represent a good feature to differentiate VBF events from QCD sourced events.

The VBF features make this channel specially interesting when there is only identified jets and we there are no additional objects in the events to rely on. For example, when the Higgs is allowed to have invisible decays, specifically as a DM production portal [2]. Figure 2 shows the Feynman diagrams for the Higgs to invisible search. This search also uses the presence of invisible particles for tagging events with large missing transverse momentum. The presence of such signal events $\mathrm{s}$ reflected in the high tails of the invariant mass of the VBF jets, as shown in Figure 3.

Beyond Higgs production many other searches have been also conducted looking for beyond SM (BSM) signatures. One very interesting search looks for compressed mass spectra in SUSY models taking profit of VBF characteristics [3]. In this search the event selection is optimized to look for events with a pair of jets with VBF characteristic plus one or zero leptons. Figure 4 displays two typical production processes for the signal events looked for in this search. Figure 5 displays the observed number of events as a function of the invariant mass of the two VBF jets for the zero leptons channel. 

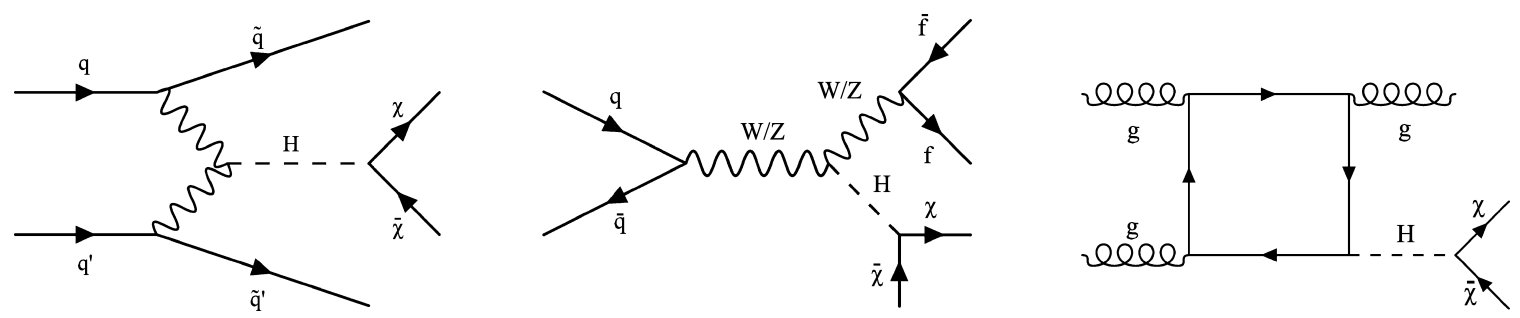

FIGURE 2: Higgs production channels with invisible decay for the Higgs to invisible search with the CMS experiment. Taken from [2].

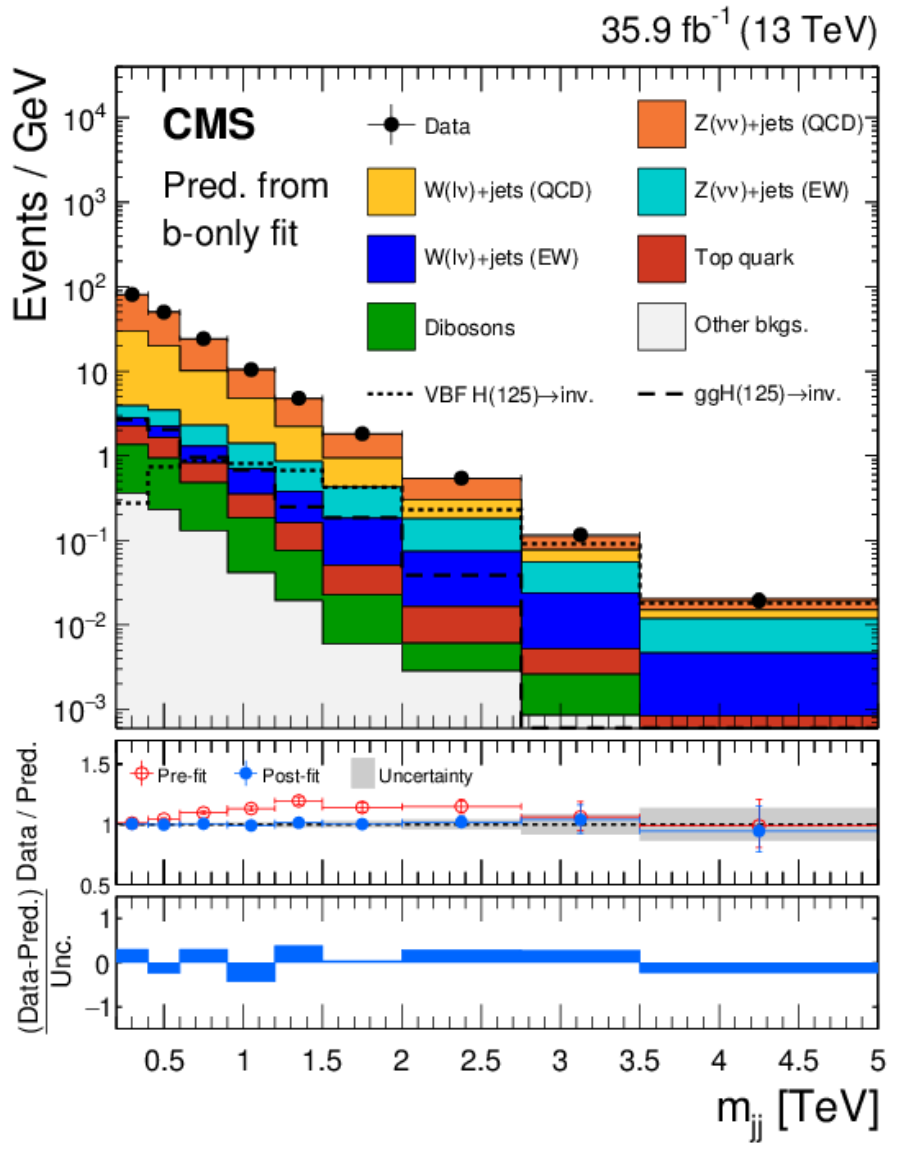

FIGURE 3: Observed events and predictions for signal in the Higgs to invisible search in the CMS experiment. Taken from [2].

With this couple of examples it can be seen that VBF is a versatile and good handle for searches at the LHC, specially interesting for the DM program.

\section{SIMPLIFIED MODELS}

The DM searches have been developing during the last years. If DM is produced in the LHC it will leave no trace of its production as DM is expected to have extremely small interactions with matter. Therefore some other particle needs to be produced with DM in order to have some observable in the ATLAS or CMS experiments. Several final states have been already explored in the LHC, for example DM production in association with jets [4], or DM production with a leptonically decaying Z boson [5], or DM produced along with a Higgs boson [6]. 

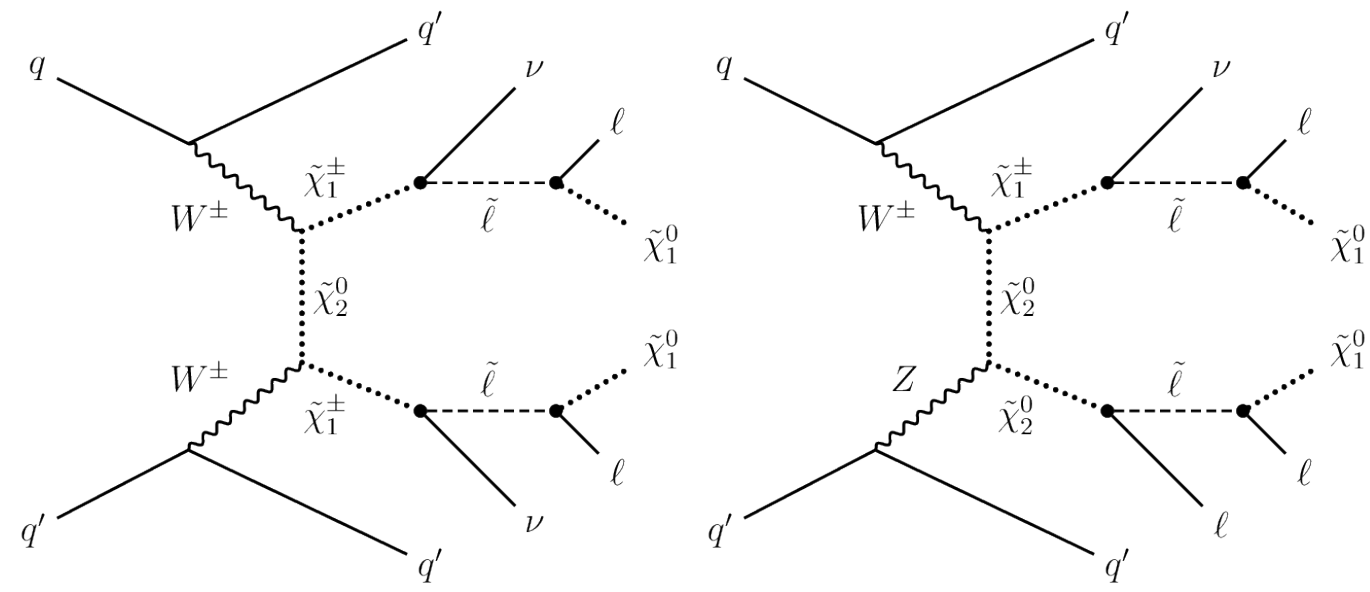

FIGURE 4: Examples of SUSY processes in VBF topology at the LHC. Taken from [3].

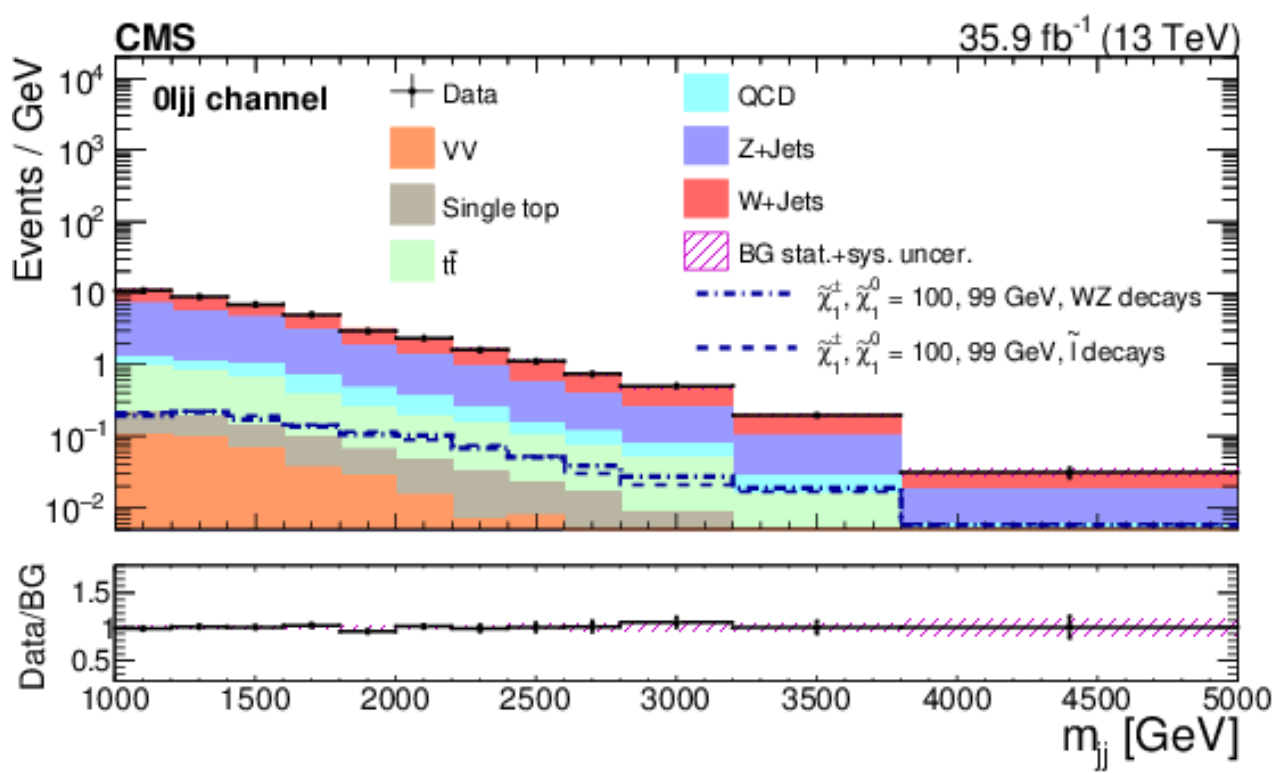

FIGURE 5: Observed events and signal predictions as a function of the invariant mass of the VBF jets for SUSY search in the zero leptons channel. Taken from [3].

However, the correct and most general modelling of DM production at colliders has remained a big challenge and different approaches have been developed. One of the most recent theoretical approaches has been called simplified models [7]. In this approach a series of models are implemented as simple extensions of the SM including a DM candidate and a mediator among the SM content and the DM candidate. In this aspect these models are minimal in content because they only include the necessary new particles in order to have DM production in colliders.

These models then provide a general framework to study the production of DM at colliders. They were developed mainly motivated by DM searches accompanied by jets, but other possibilities also arise naturally from them.

\section{VBF AND SIMPLIFIED MODELS}

We have identified that VBF DM production could be modelled in a more generic way using simplified models. Figure 6 displays two Feynman diagrams of VBF processes within the simplified models formalism.

One very interesting feature already identified from VBF processes arising at simplified models, is that typically the mediator in VBF processes have masses near to the Higgs. Within the simplified models we can vary the mediator masses and see if the kinematic behavior of the signal stays constant or varies. We have seen that for high mediator masses the eta separation between the VBF jets can significantly change and flip from high to low values. This implies that in case we could have been missing a signal in data assuming high $\eta$ separation when looking for VBF type of events. 

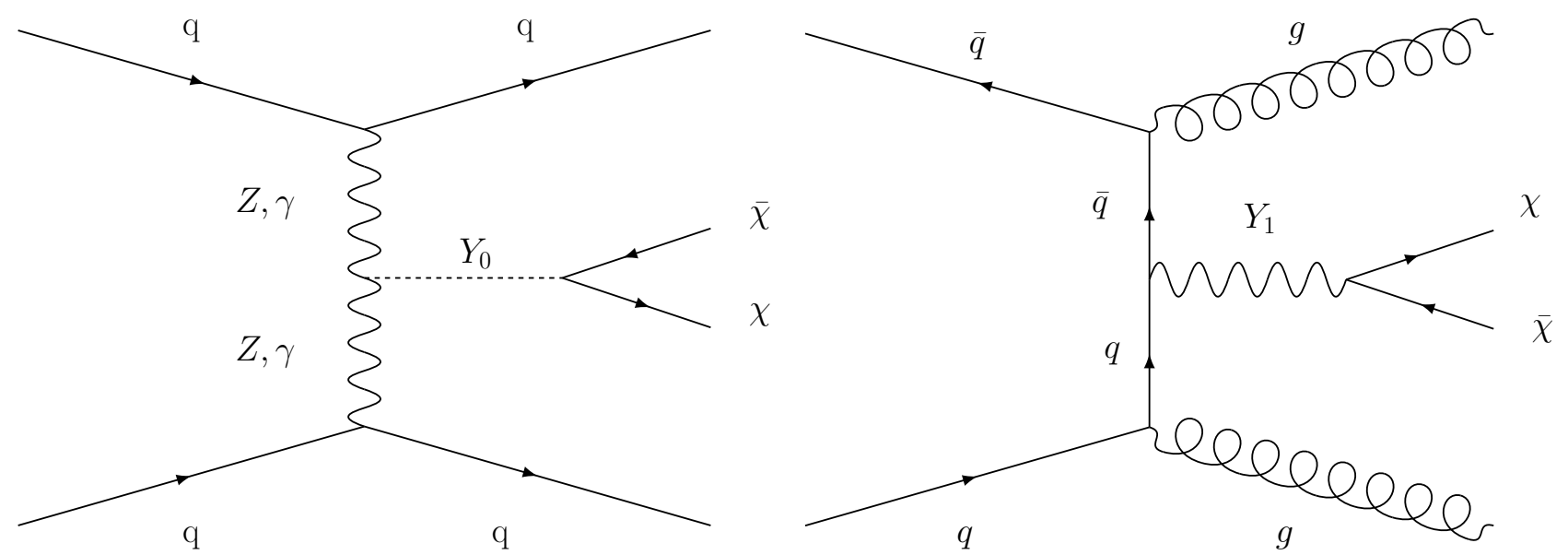

FIGURE 6: VBF processes with scalar and vector mediators in simplified models.

\section{PERSPECTIVES}

We are currently studying the VBF DM signals present in the simplified models in order to design an optimal selection for all scenarios. A special case is when the mediator mass goes up to several $\mathrm{TeV}$ and a different from usual VBF selections seems to be needed.

Aditionally we plan to compare the reach of VBF search with other searches as DM+jets in order to asses how competitive and complimentary are they.

\section{ACKNOWLEDGEMENTS}

The authors gratefully acknowledge the support of the Colombian Science Ministry and Sostenibilidad-UdeA.

\section{References}

[1] A. M. Sirunyan et al. [CMS], [arXiv:2103.06956 [hep-ex]] (2021).

[2] A. M. Sirunyan et al. [CMS], Phys. Lett. B 793 (2019), 520-551 doi:10.1016/j.physletb.2019.04.025 [arXiv:1809.05937 [hep-ex]].

[3] A. M. Sirunyan et al. [CMS], JHEP 08 (2019), 150 doi:10.1007/JHEP08(2019)150 [arXiv:1905.13059 [hep-ex]].

[4] A. M. Sirunyan et al. [CMS], Phys. Rev. D 97 (2018) no.9, 092005 doi:10.1103/PhysRevD.97.092005 [arXiv:1712.02345 [hep-ex]].

[5] A. M. Sirunyan et al. [CMS], Eur. Phys. J. C 81 (2021) no.1, 13 [erratum: Eur. Phys. J. C 81 (2021) no.4, 333] doi:10.1140/epjc/s10052-020-08739-5 [arXiv:2008.04735 [hep-ex]].

[6] A. M. Sirunyan et al. [CMS], JHEP 09 (2018), 046 doi:10.1007/JHEP09(2018)046 [arXiv:1806.04771 [hep-ex]].

[7] J. Abdallah, H. Araujo, A. Arbey, A. Ashkenazi, A. Belyaev, J. Berger, C. Boehm, A. Boveia, A. Brennan and J. Brooke, et al. Phys. Dark Univ. 9-10 (2015), 8-23 doi:10.1016/j.dark.2015.08.001 [arXiv:1506.03116 [hep-ph]]. 